Research Article

\title{
Hardware in the Loop Testing of Adaptive Inertia Weight PSO-Tuned LQR Applied to Vehicle Suspension Control
}

\author{
Joshua Sunder David Reddipogu (i) and Vinodh Kumar Elumalai ii \\ School of Electrical Engineering, Vellore Institute of Technology, Vellore 632014, India \\ Correspondence should be addressed to Vinodh Kumar Elumalai; vinothmepsg@gmail.com
}

Received 9 July 2020; Revised 7 October 2020; Accepted 15 October 2020; Published 30 October 2020

Academic Editor: Petko Petkov

Copyright (C) 2020 Joshua Sunder David Reddipogu and Vinodh Kumar Elumalai. This is an open access article distributed under the Creative Commons Attribution License, which permits unrestricted use, distribution, and reproduction in any medium, provided the original work is properly cited.

\begin{abstract}
This paper presents an adaptive inertia weight particle swarm optimization (AIWPSO) employed for solving the multiobjective weight optimization problem of LQR applied for the vehicle active suspension system (ASS). To meet the competing control objectives of ASS including the ride comfort, road handling, and suspension travel, the state feedback controller design for ASS is formulated as an optimization problem and an improved PSO is employed for finding the optimal weights of the linear-quadratic regulator (LQR). Specifically, for solving the premature convergence of the particles and imbalance between exploration and exploitation capabilities of PSO, an adaptive inertia weight that updates the velocity of the particles based on the success rate is used. The efficacy of the AIWPSO-tuned LQR is experimentally tested on a quarter-car ASS plant using the hardware in loop (HIL) testing for an uneven road surface. Experimental results highlight that, compared to conventional PSO-tuned LQR, the proposed scheme can significantly minimize the vehicle body acceleration due to irregular road profile while guaranteeing the minimum tire friction for passenger safety. The ISO 2361-1 standards adopted to evaluate the ride and health criteria substantiate that the proposed scheme reduces the vibration dose value by $25.34 \%$ for a bumpy road profile. Moreover, the cumulative power spectral density (CPSD) of vehicle body acceleration assessed in both low- and high-frequency regions manifests the significant improvement in the ride comfort.
\end{abstract}

\section{Introduction}

Active suspension systems in vehicles have gained considerable attention in both academia and industry for their potential to improve the ride comfort, road handling, and passenger safety. Compared to passive suspension systems, ASS provides better control performance in terms of minimizing the vibrations of the vehicle body due to road irregularities. In the last three decades, several control techniques, such as adaptive control [1], $H_{\infty}$ [2], sliding mode control [3], and fuzzy control $[4,5]$, have been put forward for controlling the ASS. The competing control objectives of ASS, including the ride comfort, suspension travel, and body motion, have motivated researchers to employ linear-quadratic control to realize the optimal performance without violating the constraints of the system. LQR, the cornerstone of linear-quadratic Gaussian
(LQG)/loop transfer recovery (LTR), is an optimal state feedback controller which offers several advantages including robustness, guaranteed stability, and a structured procedure which can be extended to multiple-input-multiple-output (MIMO) systems. Since LQR offers a gain margin of at least $(-6, \infty) \mathrm{dB}$ and a phase margin of $\left(-60^{\circ}, 60^{\circ}\right)$, it has been implemented in several engineering applications ranging from satellite attitude control [6], fuel cell technology [7], electric vehicles [8] to quadrotors [9]. In spite of the potential benefits of LQR, one of the major fundamental design challenges with it, for real-time applications, is the optimal choice of weighting matrices. There is no standard procedure available for the optimal selection of penalty matrices of LQR. Even though Bryson's method provides the initial choice of the penalty matrices, subsequently, it transforms into a guess-and-check approach, which is not only tedious but also time-consuming. 
Hence, the lack of knowledge of the impact of weighting matrices on the closed-loop performance has motivated researchers to explore the efficacy of swarm intelligence techniques to solve the weight selection problem of LQR.

Formulating the control performance of double inverted pendulum as a multiobjective fitness function, Wang et al. [10] put forward a multiobjective binary probability optimization algorithm for the selection of optimal weighting matrices. They adopted a binary-coding scheme to reduce the computational complexity and utilized integral absolute error (IAE) and maximum deviation (MD) as the fitness functions to improve the dynamic performance and stability of the system. Hassani and Lee [11] proposed a quantumbehaved PSO (QPSO) for deciding the optimal configuration of LQR applied to two control problems, namely, stabilizing an inverted pendulum and controlling an aircraft landing system. By introducing an aggregated dynamic weighting criterion, which dynamically combines both the hard and soft constraints with the performance objective, they validated the performance using a one-tailed $T$-test to assess whether the results are statistically significant. Ufnalski et al. [12] used PSO to optimize the gains of LQR applied to a voltage source inverter. By solving the LQR cost function minimization problem using a gradientless optimization technique, they proved that with only one penalty factor set by the designer, the full-state feedback controller with integrator could produce high-quality voltage. For solving the automatic fighter tracking problems, Tsai et al., [13] employed PSO-based variable feedback gain control. Through simulation studies, they proved that, compared to both Riccati equation-based and linear matrix inequality(LMI-) based LQR methods, PSO-based LQR offered superior performance in terms of tracking the vehicle with minimum position error. In this direction, some of the notable contributions of PSO for optimal controller design reported in the literature are power quality conditioner [14], unmanned air vehicle [15], networked control system [16], vision-based robot navigation [17], and smart structure control [18]. Even though PSO has been widely applied for LQR weight optimization, one of the common problems with the standard PSO is that while solving multimodal optimization problems, the particles may get trapped into local optima, leading to premature convergence. Moreover, as the inertia weight is kept constant, the conventional PSO fails to maintain a balance between the exploration and exploitation capabilities, resulting in suboptimal solutions. Hence, to address these two fundamental issues of PSO, in this work, we aim to utilize an adaptive inertia weight scheme in the velocity update based on the success rate of the particles. Utilizing the success rate of the particles as the feedback parameter, the AIWPSO algorithm iteratively updates the inertia weight so that it can not only avoid the premature convergence but also significantly enhance the convergence of the particles to arrive at the optimal solution.
Moreover, for assessing the consistency and repeatability of the optimization algorithms, the statistical analysis is also reported. Hence, the major contributions of this paper are twofold:

(1) The multiobjective suspension control of quarter-car ASS is formulated as an optimization problem and the gains of the state feedback controller are optimized using the AIWPSO algorithm which can enhance the convergence and offer a balance between exploration and exploitation of the particles.

(2) The efficacy of the proposed scheme to enhance the ride comfort while assuring passenger safety is experimentally tested on a laboratory scale ASS. The CPSD analysis of the vehicle body acceleration is also reported to quantitatively assess the vehicle body vibrations in both low- and high-frequency regions.

The remainder of the paper is organized as follows. Section 2 presents the AA description, dynamical equations, and performance measures. Section 3 briefly describes the problem formulation for the optimal LQR design. Section 4 details the motivations for the adaptive inertia weight scheme in the conventional PSO and the pseudocode of the AIWPSO. Section 5 explains the statistical analysis of the optimization algorithms and the experimental validation of the proposed control scheme. Section 6 gives the concluding remarks of the paper.

\section{Active Suspension System}

2.1. Mathematical Modeling. Figure 1 shows the schematic diagram of a quarter-car model with ASS, which depicts the two-mass-spring damper system. The ASS consists of a sprung mass $M_{s}$ and an unsprung mass $M_{\mathrm{us}}$, representing the vehicle body and the wheel assembly in the quarter-car model. Both the sprung mass and unsprung mass are supported by springs and dampers. The stiffness of the tire with respect to the road is modeled by the spring $K_{\mathrm{us}}$ and the damper $B_{\mathrm{us}}$. Similarly, the bodyweight over the tire is balanced by the spring $K_{s}$ and the damper $B_{s}$. To counteract the vertical forces created due to an uneven road surface, the system contains an electrical actuator positioned between the vehicle body and the wheel assembly [19-21]. The suspension controller drives this actuator based on the vehicle body acceleration and suspension travel. Using the force balance equation based on Newton's second law, we obtain the following equation of motion of ASS:

$$
\begin{array}{cl}
M_{\mathrm{us}} \ddot{Z}_{\mathrm{us}} & =-B_{s} \dot{z}_{\mathrm{us}}-B_{\mathrm{us}} \dot{z}_{\mathrm{us}}-F_{c}+B_{\mathrm{us}} \dot{z}_{r} \\
& -\left(Z_{\mathrm{us}}-Z_{s}\right) K_{s}-\left(Z_{\mathrm{us}}-Z_{s}\right) K_{\mathrm{us}}, \\
M_{s} \ddot{Z}_{s} & =B_{s} \dot{z}_{\mathrm{us}}+F_{c}-B_{s} \dot{z}_{s}-\left(Z_{s}-Z_{\mathrm{us}}\right) K_{s} .
\end{array}
$$



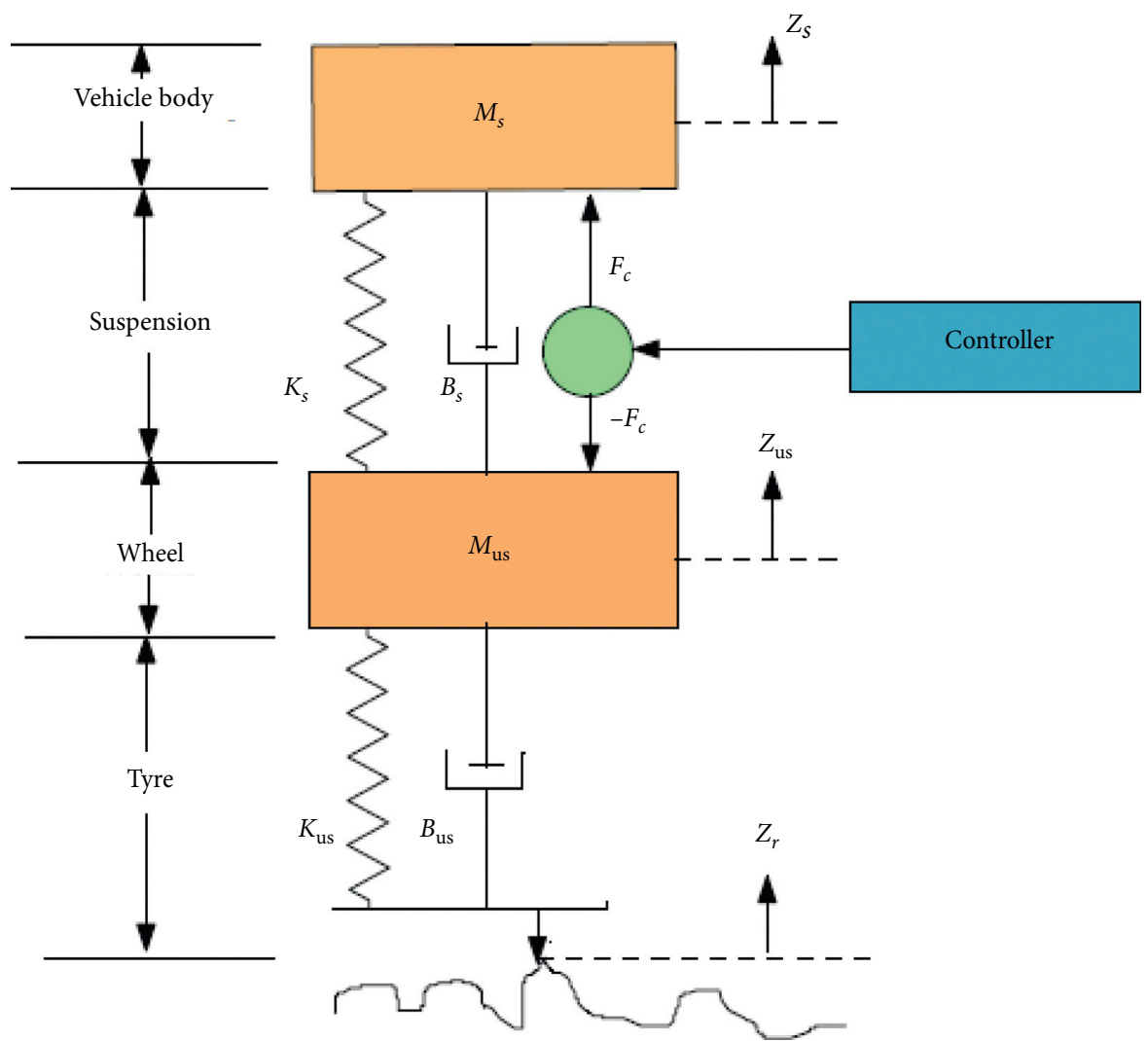

FIGURE 1: Free body diagram of the quarter-car ASS.

For obtaining the state-space model of the system, we consider the following state and input vectors: $x=\left[Z_{s}-Z_{\text {us }}, \dot{Z}_{s}, Z_{\text {us }}-Z_{r}, \dot{Z}_{\text {us }}\right]^{\top}$, and $u=F_{c}$ :

$\dot{x}(t)=\left[\begin{array}{cccc}0 & 1 & 0 & 1 \\ -\frac{K_{s}}{M_{s}} & -\frac{B_{s}}{M_{s}} & 0 & \frac{B_{s}}{M_{s}} \\ 0 & 0 & 1 & 0 \\ \frac{K_{s}}{M_{\mathrm{us}}} & \frac{B_{s}}{M_{\mathrm{us}}} & -\frac{K_{\mathrm{us}}}{M_{\mathrm{us}}} & -\frac{B_{s}+B_{\mathrm{us}}}{M_{\mathrm{us}}}\end{array}\right] x(t)+\left[\begin{array}{c}0 \\ \frac{1}{M_{s}} \\ 0 \\ -\frac{1}{M_{\mathrm{us}}}\end{array}\right] u(t)$,

$y(t)=\left[\begin{array}{cccc}1 & 0 & 0 & 0 \\ -\frac{K_{s}}{M_{s}} & -\frac{B_{s}}{M_{s}} & 0 & \frac{B_{s}}{M_{s}}\end{array}\right] x(t)+\left[\begin{array}{c}0 \\ -\frac{1}{M_{s}}\end{array}\right] u(t)$.

The four states of the ASS are $Z_{s}-Z_{\mathrm{us}}, \dot{Z}_{s}, Z_{\mathrm{us}}-Z_{r}$, and $\dot{Z}_{\text {us }}$, which are the suspension deflection, the vehicle body vertical velocity, the tire deflection, and the tire vertical velocity, respectively. The control force $F_{c}$ created by the electrical actuator acts as the control input, and the output variables of interest are the vehicle body acceleration $\ddot{Z}_{s}$ and the suspension deflection. Table 1 gives the plant parameters of the ASS workstation.
TABLE 1: Quarter-car active suspension system plant parameters.

\begin{tabular}{lcc}
\hline Symbol & Description & Value \\
\hline$M_{s}$ & Sprung mass & $2.45 \mathrm{~kg}$ \\
$M_{\text {us }}$ & Unsprung mass & $1 \mathrm{~kg}$ \\
$B_{s}$ & Suspension damping constant & $7.5\left(\mathrm{~N}-\mathrm{s}^{2} / \mathrm{m}\right)$ \\
$B_{\text {us }}$ & Tire damping constant & $5\left(\mathrm{~N}-\mathrm{s}^{2} / \mathrm{m}\right)$ \\
$K_{s}$ & Suspension stiffness constant & $490 \mathrm{~N} / \mathrm{m}\left(\mathrm{N}-\mathrm{s}^{2} / \mathrm{m}\right)$ \\
$K_{\text {us }}$ & Tire stiffness constant & $1020 \mathrm{~N} / \mathrm{m}$ \\
- & Suspension travel range & $\left(\mathrm{N}-\mathrm{s}^{2} / \mathrm{m}\right)$ \\
- & Accelerometer sensitivity & $9.8 \times 10^{-2} \mathrm{~m}$ \\
- & Suspension encoder resolution & $942 \times 10^{-6} \mathrm{~m} / \mathrm{count}$ \\
- & Suspension motor torque & $0.115 \mathrm{~N}-\mathrm{m} / \mathrm{A}$ \\
\hline
\end{tabular}

2.2. Performance Measures. The conflicting performance requirements of ASS, which need to be considered while designing a feedback controller, are as follows:

(1) Ride comfort: the primary objective of the vehicle suspension system is to improve passenger comfort by reducing the vehicle body acceleration $\ddot{Z}_{s}$, arising from an uneven road surface.

(2) Suspension travel: while ensuring the minimum level of vehicle body acceleration, the ASS has to ensure that the suspension deflection is regulated within the permissible range, thereby preventing any structural damage: 


$$
\left|Z_{s}-Z_{\text {us }}\right| \leq Z_{\max }
$$

where $Z_{\max }$ is the maximum suspension deflection.

(3) Road handling: to guarantee passenger safety, it is paramount to ensure that the wheel assembly maintains firm contact with the road. Therefore, the static load of the tire has to be greater than its dynamic load as given as follows:

$$
\left|K_{s}\left(Z_{\mathrm{us}}-Z_{r}\right)\right| \leq\left(M_{s}+M_{\mathrm{us}} g\right) .
$$

To realize a feedback controller which can solve these conflicting control objectives, we aim to synthesize an optimal state feedback controller based on linear-quadratic theory. Then, we experimentally assess the performance of the optimal state feedback controller to suppress the vehicle body vibration while guaranteeing passenger safety. In the following section, we present the problem formulation based on the LQ theory and subsequently discuss the variant of the PSO algorithm to solve the weight optimization problem of LQR.

\section{Problem Formulation}

For a linear time-invariant (LTI) system, the linear-quadratic optimal control problem is as follows: given the system

$$
\begin{aligned}
& \dot{x}(t)=A x(t)+B u(t), \quad t \geq 0, x(0)=x_{0}, \\
& y(t)=C x(t)+D u(t), \quad t \geq 0,
\end{aligned}
$$

with the initial condition $x(0)=x_{0}$, we find the optimal control input $u^{*}(t)$ that can regulate the system dynamics to the desired state by minimizing the following quadratic objective function:

$$
J\left(u^{*}\right)=\int_{0}^{\infty} x^{T}(t) Q x(t)+u^{T}(t) R u(t) \mathrm{d} t
$$

where $Q=Q^{T}$ is the positive semidefinite state penalty matrix, and $R=R^{T}$ is the positive definite control input penalty matrix. To make the cost function quadratic, the penalty matrices, also called weighing matrices of LQR, are chosen to be diagonal matrices. The number of states decides the order of the $Q$ matrix, and the number of controls input dictates the order of $R$ matrix. With the assumption that the pair $(A, B)$ is controllable and the pair $(A, C)$ is detectable, LQR implements the following optimal state feedback law:

$$
u=-K x(t)
$$

where $K$ is the optimal state feedback gain determined based on the following Lagrange multiplier based optimization approach:

$$
K=R^{-1} B^{T} P
$$

The transformation matrix, $P$, is found by solving the following algebraic Riccati equation (ARE):

$$
A^{T} P+P A+Q-\mathrm{PBR}^{-1} B^{T} P=0 .
$$

The tradeoff between the control effort and the speed of response is largely decided by the choice of the weighting matrices. However, because of the lack of knowledge on the relation between the closed-loop performance and quadratic weights, one of the challenges associated with the LQR design is the technique for selecting the weighting matrices. Even though Bryson's rule provides the initial choice of $Q$ and $R$ matrices, eventually, it is reduced to a trial and error approach, which is both time-consuming and tedious. Hence, to solve the weight selection problem of LQR, we formulate the performance measures as a cost function and discuss an adaptive inertia weight-based PSO algorithm in the following section for solving the weight optimization problem of LQR.

\section{AIWPSO for LQR Weight Optimization}

PSO, a stochastic population-based optimization algorithm, is a swarm intelligence technique proposed by Kennedy and Eberhart by being inspired by the social behavior of birds and fish [22]. Utilizing the group of particles that represent the potential solutions for the given optimization problem, PSO moves the particles around the $n$-dimensional hyperspace to find the global optimal solution by minimizing the fitness function. The particles use both their personal best and the neighbors' best performances to reach the optimal solution. Figure 2 illustrates the velocity update mechanism in a standard PSO. The position and velocity of the particles are updated during every iteration based on the following equations:

$$
\begin{aligned}
v_{k}^{d}(t+1)= & w v_{k}^{d}(t)+c_{1} \operatorname{rand}_{1}\left(x_{\text {pbest }_{k}}^{d}-x_{k}^{d}(t)\right) \\
& +c_{2} \operatorname{rand}_{2}\left(x_{\text {gbest }_{k}}^{d}-x_{k}^{d}(t)\right), \\
x_{k}^{d}(t+1)= & x_{k}^{d}(t)+v_{k}^{d}(t+1),
\end{aligned}
$$

where $c_{1}$ and $c_{2}$ are the cognitive acceleration constants, which influence the learning rate of the particles, $w$ is the inertia weight, and $\operatorname{rand}_{1}$ and $\operatorname{rand}_{2}$ are the uniformly distributed random vectors. pbest indicates the local optimum and gbest represents the global optimum. One of the major advantages of PSO is that it is a derivative-free optimization algorithm which is less sensitive to the type of cost function. Furthermore, the convergence of PSO is not affected by the initial conditions of the particles and the size of the nonlinearity problem [23]. Hence, PSO has found applications in several areas such as aerospace [24], antenna design [25], and power systems [26]. However, the two standard limitations of PSO have restricted its use in solving several real-world optimization problems. Firstly, while solving multimodal optimization problems, the particles may get trapped into local optima, leading to premature convergence or loss of diversity. Secondly, the PSO fails to draw a balance between the exploration and exploitation capabilities of the particles, which result in suboptimal solutions. Hence, to address these problems, several variants of PSO have been put forward in the last three decades. 


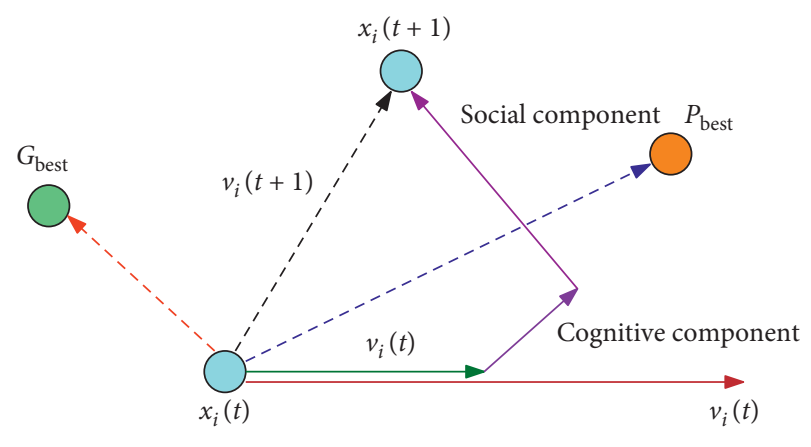

FIgURE 2: PSO velocity diagram.

In order to improve the convergence, Eberhart and Shi [27] initially proposed the idea of making the inertia weight in the velocity update function dynamic instead of constant as in the case of conventional PSO. Subsequently, several adaptation mechanisms in the inertia weight have been introduced in the PSO including random inertia weights, time-varying inertia weights, and adaptive inertia weights. For a detailed survey on the adaptive mechanisms, the readers can refer to [28]. It is important to mention that the major focus of this paper is to explore the adaptive inertia weight mechanism to enhance the convergence and improve the exploration and exploitation capabilities of the particles. Exploitation is the capability of the algorithm to further refine the best solution found so far, by inspecting a small neighborhood of that solution. In contrast, exploration indicates the potential of the algorithm to leave the current optimal solution and explore better solutions. Putting large emphasis on the exploration results in pure random search in the search space. On the other hand, overly focusing on exploitation leads to premature convergence. Hence, it is important to draw a balance between the exploration and exploitation capabilities in order to take advantage of the ability of the algorithm to not only find all possible solutions but also refine the solutions to reach the global optimum without compromising on convergence. In order to address these issues, we aim to employ the adaptive inertia weight scheme, which uses the success rate of the particles, for updating the velocity of the particles. Figure 3 illustrates the adaptive inertia weight mechanism reinforced PSO. The basic methodology behind the velocity update is as follows. While solving the optimization problem, if the fitness value of the current iteration is more than that of the previous iterations, the success rate (SR) is set to 1 ; else, SR is set to zero. Therefore, the SR can be represented as follows:

$$
\mathrm{SR}_{i}= \begin{cases}1, & \mathscr{F}<\mathscr{F}_{\text {pbest }}{ }_{i} \\ 0, & \mathscr{F} \geq \mathscr{F}_{\text {pbest }}{ }_{i}\end{cases}
$$
follows:

The percentage of success (PS) is determined using SR as

$$
\mathrm{PS}=\frac{\sum_{i=1}^{N} \mathrm{SC}_{i}}{N},
$$

where $N$ indicates the number of particles. A high value of PS indicates that the particle's current position is far away from the optimum point and the swarm is slowly approaching the optimum. In contrast, a low PS value indicates that the particles are moving around the optimum without much improvement. Hence, it is reasonable to select the inertia weight $w$ as a function of PS. The adaptive inertia weight factor (AIWF) chosen as a linear function which maps the values of PS to the range of $w$ is given by

$$
w(t)=\left(w_{\max }-w_{\min }\right) \mathrm{PS}+w_{\min } .
$$

The range of the inertia weight is $[0,1]$. Utilizing the success rate of the particles as a feedback parameter, AIWPSO determines the state of the particles and enhances the convergence by adaptively updating the inertia weights.

To investigate the impact of the inertia weight on the local and global search capabilities of PSO, let us consider two different situations as follows. First, as shown in Figure 4(a), two particles, moving toward the optimum, are close to each other but located far from the global optimum. If we neglect the effect of personal and global best positions in equation (10), then a $w$ value greater than 1 accelerates the particle with maximum velocity and a value of $w$ less than 1 decelerates the particle velocity to 0 . In the second situation, depicted in Figure 4(b), the global and local best positions are close to the optimum and the particles can explore the small region, also called the improvement region, and enhance the quality of best solutions $[29,30]$. If the velocity of the particle is high, the particle will continue to oscillate around the improvement region with less probability of achieving a better solution. Hence, in this situation, the velocity of the particle can be minimized by reducing the inertia weight. Algorithm 1 presents the pseudocode of the AIWPSO algorithm to solve the dynamic optimization problem.

Figure 5 shows the proposed AIWPSO-based LQR design for multiobjective suspension control. The suspension travel and the tire displacement are measured using optical encoders, and the suspension velocity and tire velocity are obtained using the following second-order derivative filter with the natural frequency of oscillation being $\omega_{n}=15.7(\mathrm{rad} / \mathrm{sec})$.

$$
H(s)=\frac{\omega_{n}^{2}}{s^{2}+2 \zeta \omega_{n} s+\omega_{n}^{2}} .
$$

Since the dynamics of ASS is modeled as a fourth-order system, the $Q$ matrix should be a $4 \times 4$ positive semidefinite matrix. In addition, as the system receives one control input $F_{c}$ from the actuator, the input penalty $R$ is a scalar. Hence, the resultant cost function to be optimized is

$$
J=\int_{0}^{\infty}\left(q_{1} x_{1}^{2}+q_{2} x_{2}^{2}+q_{3} x_{3}^{2}+q_{4} x_{4}^{2}+R_{1} u_{1}^{2}\right) \mathrm{d} t,
$$

where $q_{1}, q_{2}, q_{3}$, and $q_{4}$ are the weights of suspension travel, suspension velocity, tire deflection, and tire velocity, respectively. Similarly, $R_{1}$ is the weight factor for the control force generated by the actuator. For optimizing the weights using the PSO algorithm, the following integral square error (ISE) is considered as the fitness function: 


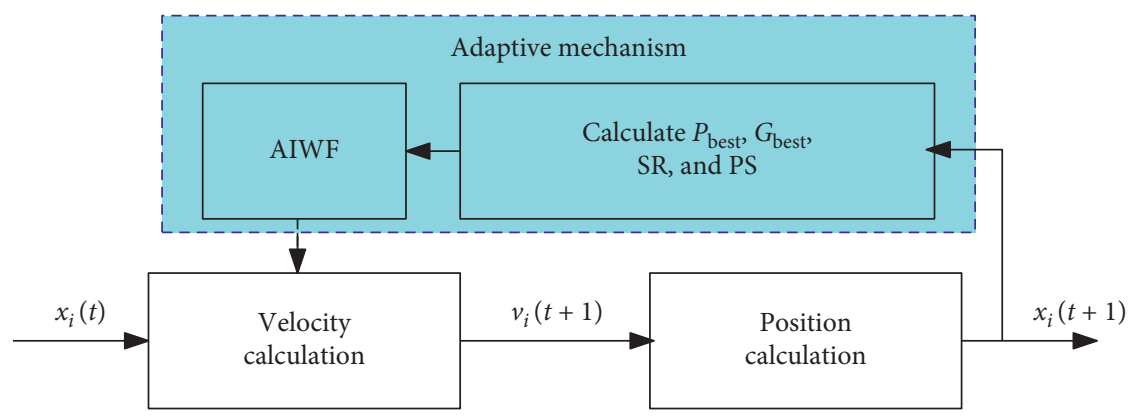

FIgURE 3: AIWPSO schematic diagram.

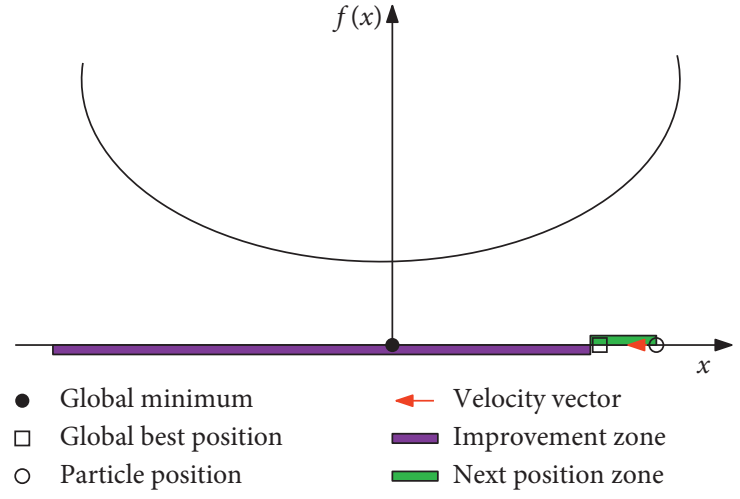

(a)

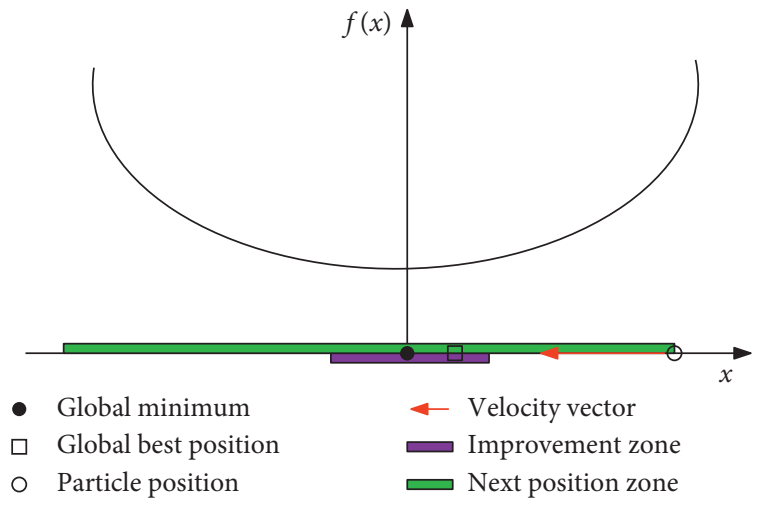

(b)

FIgURE 4: AIWPSO Scenarios. (a) Near global solution. (b) Far from global solution.

(1) Initialize the population size $N$, the dimensions $D$, maximum no of iterations $T_{\max }$, inertia weight $\omega$, location boundary $\left[x_{\min }^{d}, x_{\max }^{d}\right]$, and velocity boundary $\left[v_{\min }^{d}, v_{\max }^{d}\right]$.

(2) for $i=1$ to $T_{\max }$

(3) Initialize the success rate (SR) $=0$

(4) for $i=1$ to $N$

(5) Compute the fitness of the particles $f=\int e^{2}(t) \mathrm{d} t$

(6) if $f<f_{\text {Pbest }_{i}}$

(7) $\mathrm{SR}=\mathrm{SR}+1$

(8) $\quad f_{\text {Pbest }_{i}} \leftarrow f$

(9) $\quad x_{\text {Pbest }_{i}} \leftarrow x_{i}$

(10) end

(11) if $f<f_{\text {Gbest }_{i}}$

(12) $\quad f_{\text {Gbest }_{\mathrm{i}}} \leftarrow f$

(13) $\quad x_{\text {Gbest }_{i}} \leftarrow x_{i}$

(14) end

(15) for $i=1$ to $D$

(16) Update the velocity and position of the particle

(17) $v_{k}^{d}(t+1)=w v_{k}^{d}(t)+c_{1} \operatorname{rand}_{1}\left(x_{\text {pbest }_{k}}^{d}-x_{k}^{d}(t)\right)+c_{2} \operatorname{rand}_{2}\left(x_{\text {gbest }_{k}}^{d}-x_{k}^{d}(t)\right)$

(18) $x_{k}^{d}(t+1)=x_{k}^{d}(t)+v_{k}^{d}(t+1)$

(19) end

(20) Calculate the percentage of success using 14

(21) Update the inertia weight using 15

(22) end

(23) end 


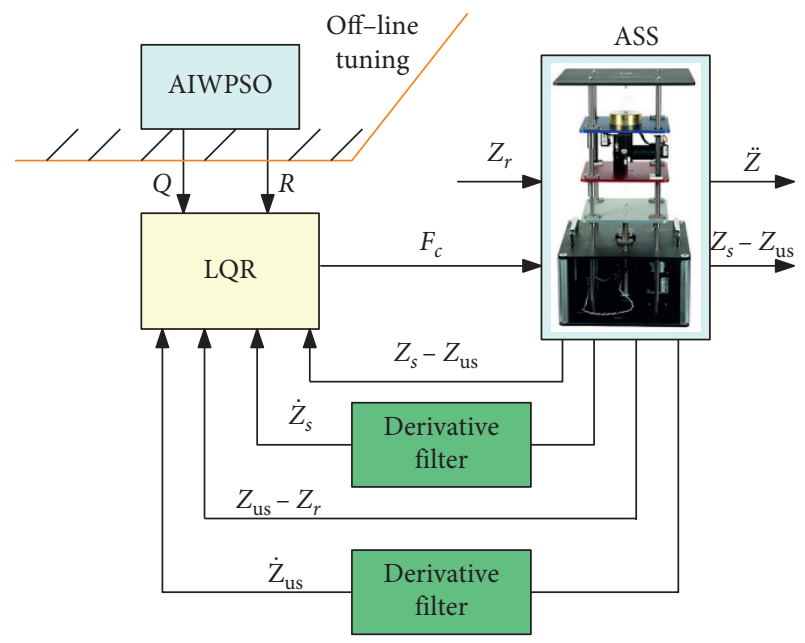

FIgure 5: Proposed AIWPSO-tuned LQR for quarter-car ASS.

$$
F=\int_{0}^{\infty} e^{2}(t) \mathrm{d} t .
$$

The objective is to find the optimal weights of the LQR and determine the state feedback gain vector $K=\left[k_{1}, k_{2}, k_{3}, k_{4}\right]$ such that the controller can meet the competing performance objectives of ASS.

\section{Experimental Results and Discussion}

Figure 6 shows the ASS workstation, which consists of three plates on top of each other. The top plate, suspended with two springs over the middle plate, emulates the vehicle body and contains an accelerometer to acquire the acceleration of the vehicle body with respect to plant ground. A servomotor placed between the top and middle plates acts as an actuator to counteract the vertical forces created due to an uneven road profile. The middle plate maintains contact with the bottom plate through a spring. The bottom plate, which resembles the wheel of the quarter car, is connected to a DC motor to generate the road excitation in the system. The rotational motion created due to the torque from the motor is translated into linear motion using the lead screw and gearing mechanism for creating different road profiles.

Figure 7 shows the ASS experimental test-bed, which consists of a quarter-car ASS workstation interfaced with a computer, power amplifiers, and an 8-channel USB-based data acquisition module (DAQ). To measure the suspension deflection and tire displacement, the ASS contains three optical encoders, which have a resolution of 4096 counts/ revolution in quadrature mode. Similarly, to acquire the vehicle body acceleration relative to the ground, the top plate is attached with an accelerometer with a range of $\pm 10 \mathrm{~g}$. The power amplifiers, which drive the servomotors, can offer a regulated supply of $\pm 10 \mathrm{~V}$ at $3 \mathrm{~A}$. The DAQ board, which has a resolution of 12 bits, acquires signals at a sampling rate of $500 \mathrm{~Hz}$. For HIL testing, the control algorithm implemented in Simulink is interfaced with the ASS workstation using the Quanser real-time control prototype software. Figure 8 shows the suspension control implementation in Simulink.
Table 2 gives the parameters of the PSO and AIWPSO for optimizing the weights of LQR. From a control standpoint, the number of parameters to be optimized is 5, which include the four states and the control input. Hence, the dimension of the particles is initialized to 5, and the range of weights for the $Q$ matrix is assigned from 0 to 500 . Moreover, to guarantee a feasible control input, the range of the input penalty factor is set between 0 and 10, keeping the servomotor voltage constraint. In both conventional PSO and AIWPSO, the number of particles is initialized to 30 , and the iteration based termination condition is chosen with the maximum number of iterations being 500 .

Figure 9 shows the convergence of the fitness function for both PSO and AIWPSO. It is evident from the fitness plot that the AIWPSO converges faster than the conventional PSO. To be specific, PSO takes 60 iterations to converge whereas the AIWPSO takes only 30 iterations to converge to the minimum fitness value. To highlight the improvement in convergence, we also illustrate the convergence of the particles in each domain as a 3D scatter plot. Figure 10 shows the initial random distribution of the particles, and Figure 11 depicts the convergence after 100 iterations. Likewise, from the convergence of the particles for every 100 iterations shown in Figures 12-15, we can discern that unlike the conventional PSO, which takes around 500 iterations in all 5 domains to converge, the AIWPSO converges to the optimal solutions in about 200 iterations. The main reason for the faster convergence can be attributed to the adaptive inertia weights which are dynamically adjusted based on the success rate of the particles.

Since PSO is a stochastic optimization algorithm, to assess the accuracy and repeatability, the experiments are conducted for 10 trials to statistically analyze the solutions of both conventional PSO and AIWPSO. Table 3 gives statistical measures such as minimum, maximum, standard deviation, and range of the optimal solutions. It is evident that the range and mean fitness values of AIWPSO is significantly less than those of PSO. Hence, the proposed LQR optimization scheme has better precision and consistency in achieving the optimal solution. Table 4 gives the optimized state and input penalty matrices of LQR from both algorithms.

The controller performance is tested for two classes of road inputs, namely, a trapezoidal road profile and a bump road profile. Firstly, a trapezoidal road profile with an amplitude of $20 \mathrm{~cm}$ at a frequency of $0.5 \mathrm{rad} / \mathrm{sec}$ is generated using the servomotor and lead screw arrangement, which is positioned at the bottom of the ASS plant. From Figure 16, which shows the suspension travel response of both PSO and AIWPSO, we can note that compared to PSO, the AIWPSO optimized LQR results in better steadystate response and regulates the suspension movement close to the road profile to avoid the tire structural damage. Figure 17, which illustrates the tire deflection, highlights that tire displacement is precisely governed to match the road profile in AIWPSO so that passenger safety is guaranteed. Moreover, to assess the passenger ride comfort, the vertical vehicle body acceleration is shown in Figure 18. Clearly, the proposed scheme outperforms the 


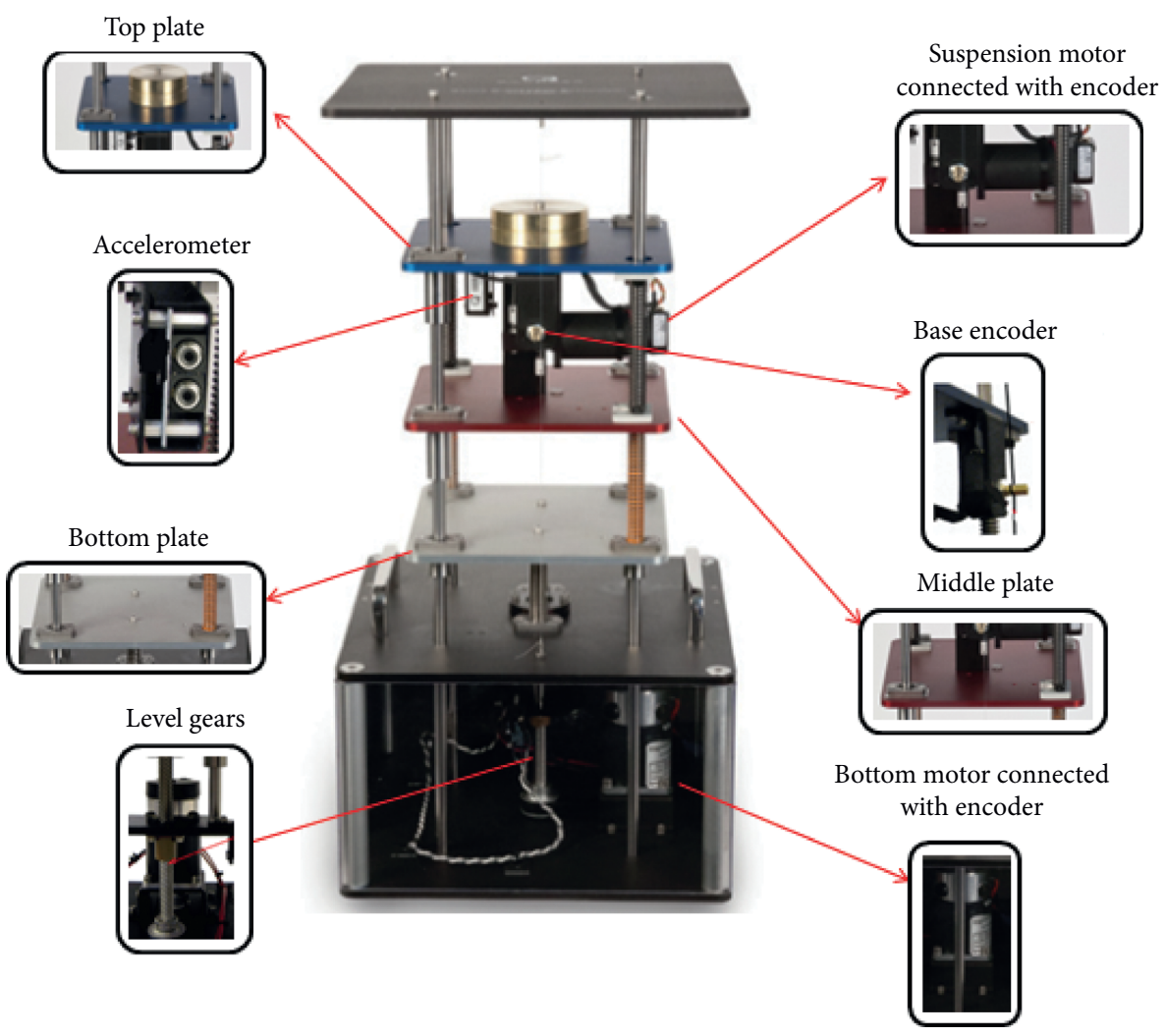

FIGURE 6: Quarter-car ASS workstation.

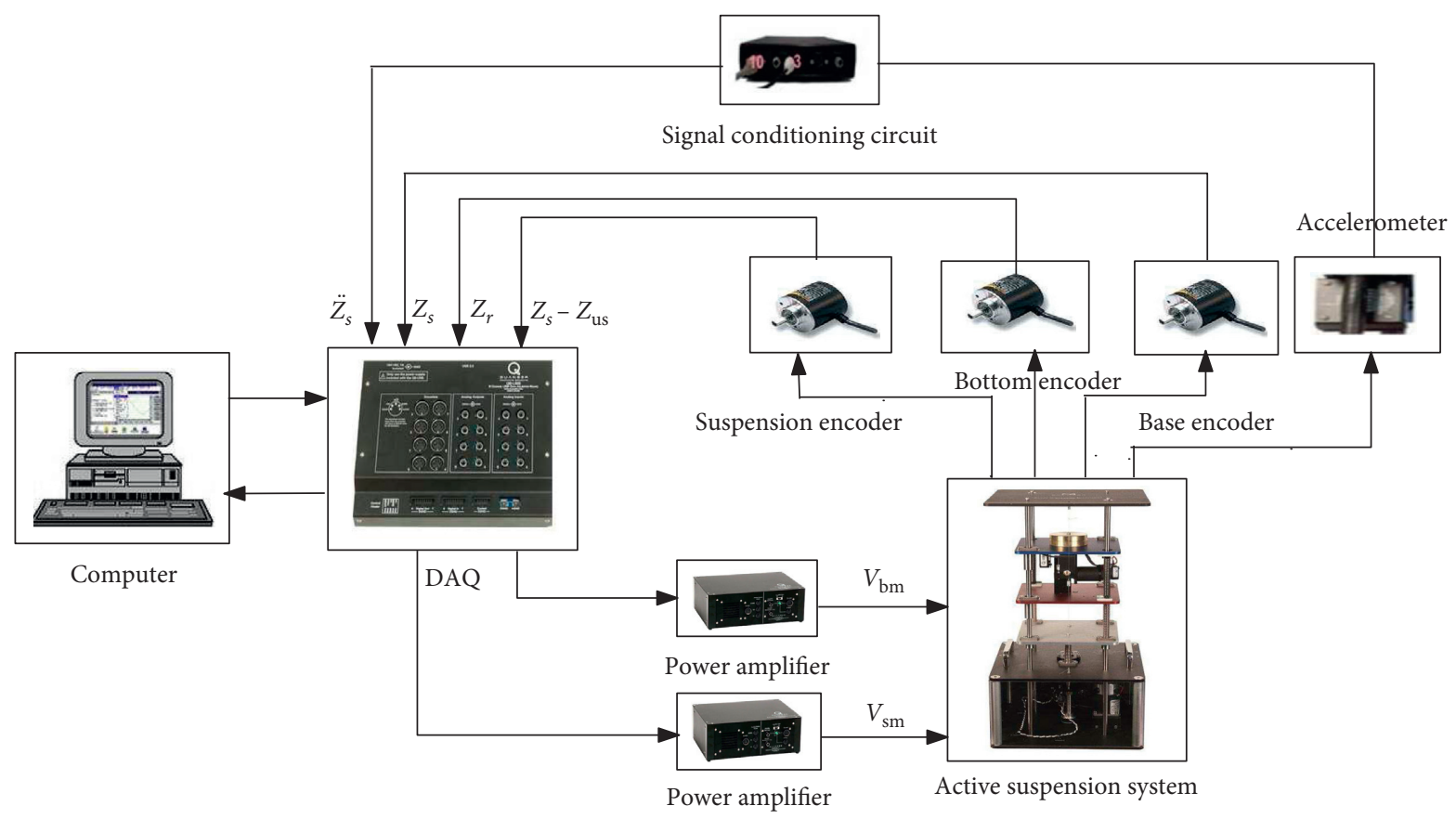

Figure 7: Experimental test-bed.

conventional PSO-tuned LQR and significantly improves the ride comfort. It is also important to assess the vibration of the vehicle body in both low- and high-frequency domains. Hence, the CPSD of the vehicle body acceleration is computed and shown in Figure 19. We can note that both during the low- and high-frequency spectra, the proposed scheme significantly minimizes the vibration of the chassis. Finally, the control effort generated through the electrical 


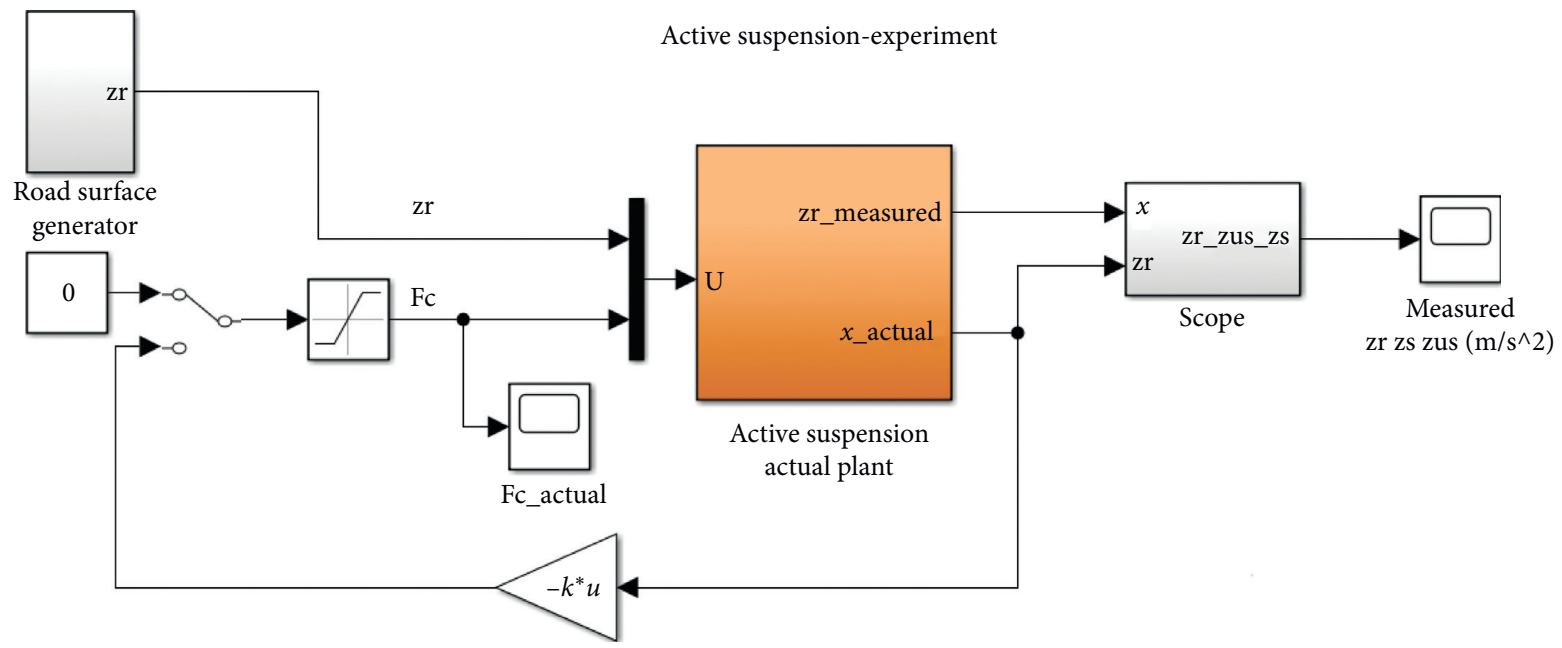

FIgURE 8: HIL implementation in Simulink.

TABLE 2: Parameters of PSO and AIWPSO algorithms.

\begin{tabular}{lccc}
\hline Parameter & Notation & PSO & AIWPSO \\
\hline Particles & $P$ & 30 & 30 \\
Iteration & $i$ & 500 & 500 \\
Dimension & $d$ & 5 & 5 \\
Inertia weight & $w$ & 0.9 & AIWF \\
Cognitive factors & {$\left[c_{1} c_{2}\right]$} & {$\left[\begin{array}{lll}0.9 & 1.2\end{array}\right]$} & 0.2 \\
\hline
\end{tabular}

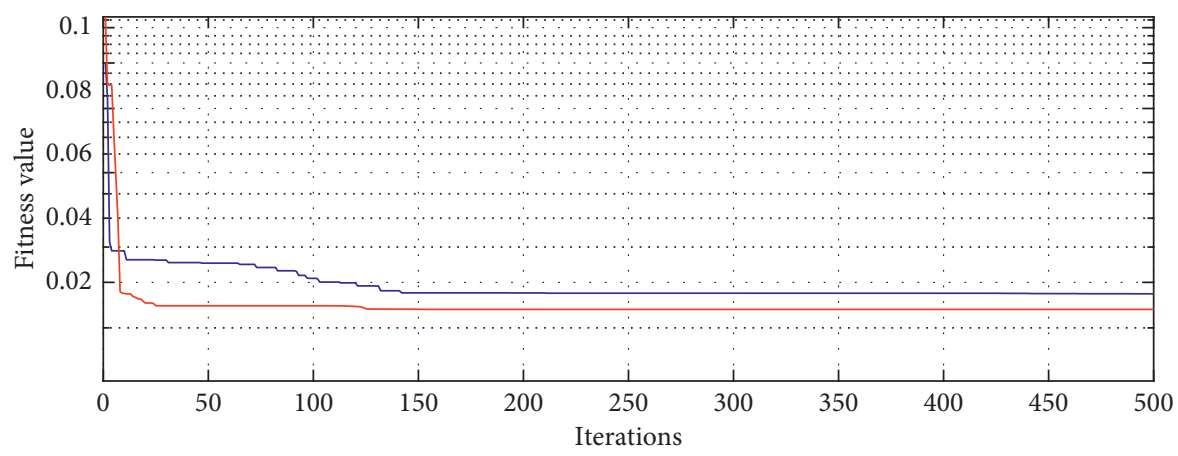

- PSO

- AIWPSO

FIgURE 9: Fitness function plot.

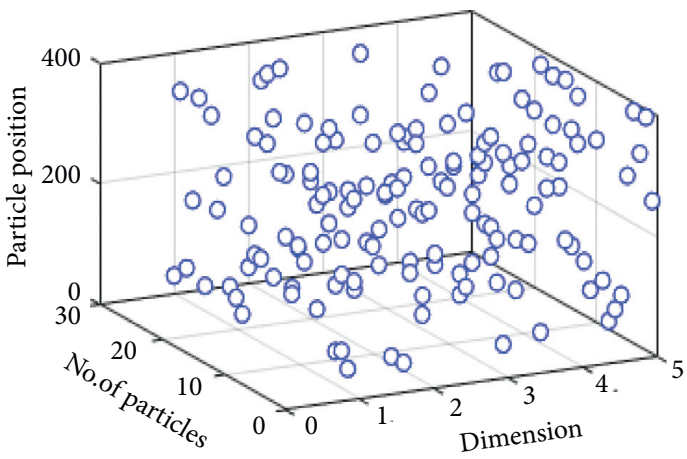

(a)

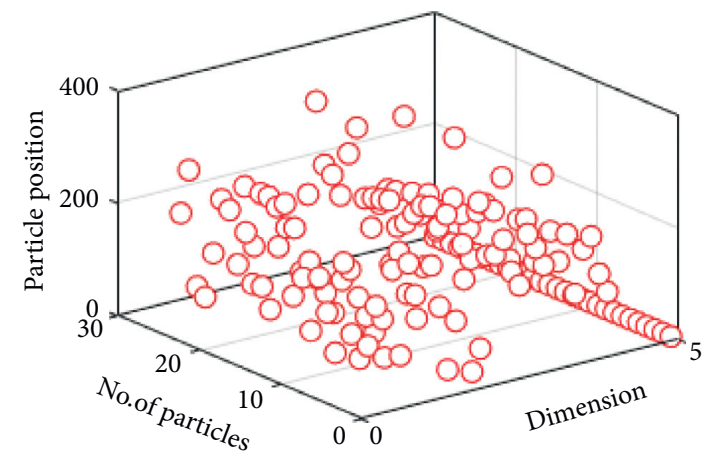

(b)

FIgURE 10: Random Initialization of particles. (a) PSO. (b) AIWPSO. 


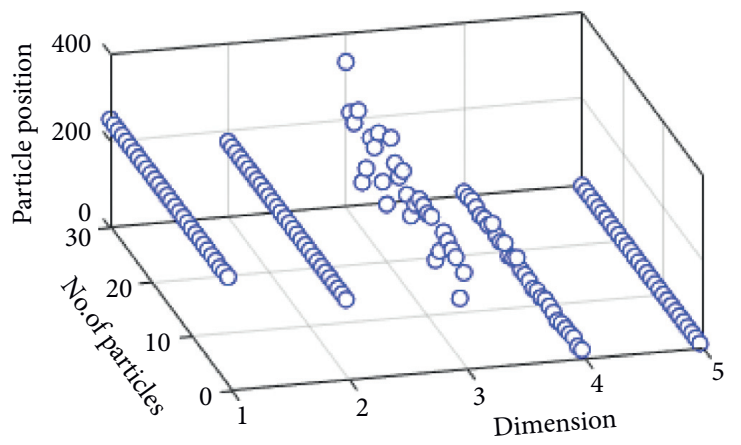

(a)

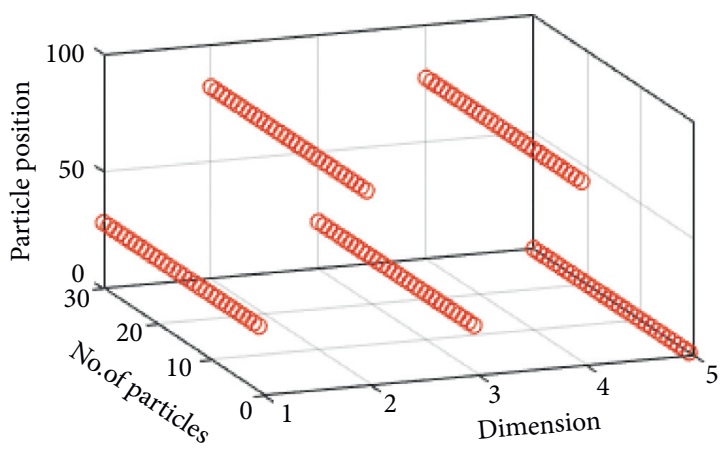

(b)

Figure 11: Particle convergence after 100 iterations. (a) PSO. (b) AIWPSO.

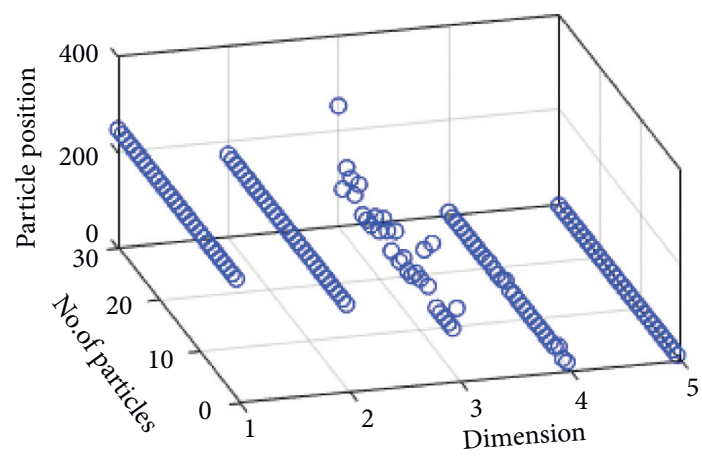

(a)

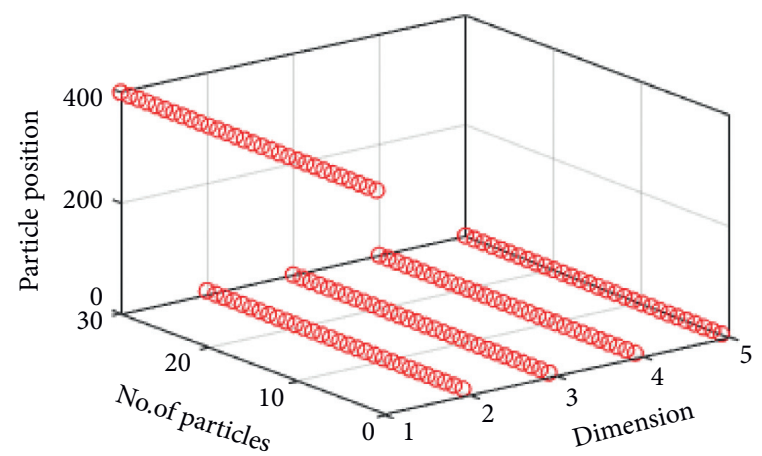

(b)

FIgure 12: Particle convergence after 200 iterations. (a) PSO. (b) AIWPSO.

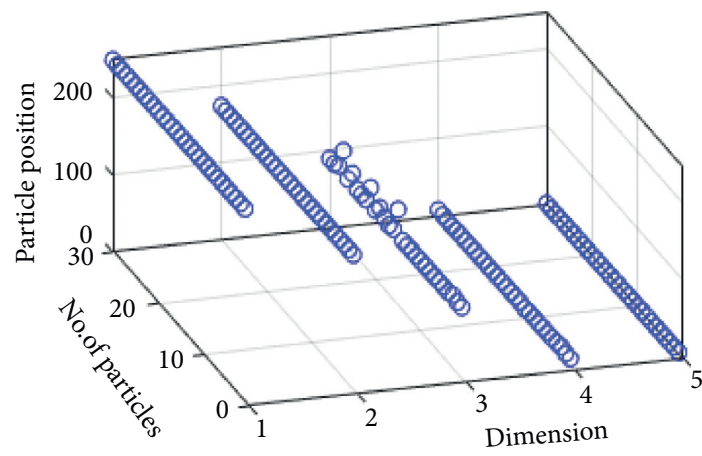

(a)

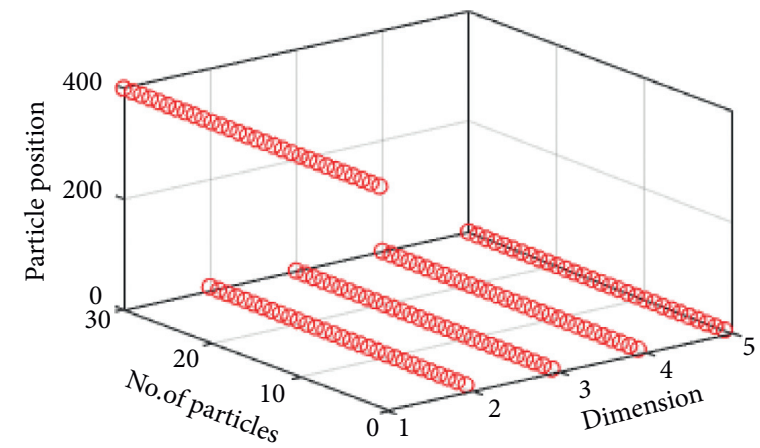

(b)

Figure 13: Particle convergence after 300 iterations. (a) PSO. (b) AIWPSO.

actuator, as illustrated in Figure 20, proves that the force is restricted within $\pm 10 \mathrm{~N}$ for a feasible control input.It is also important to test the performance of the system for the speed breaker scenario. Hence, a pattern consisting of a series of half-sine waves is generated which emulates a speed breaker, and the performance of the controller in terms of vehicle body acceleration and suspension travel is illustrated in Figures 21 and 22, respectively. Figure 23, which shows the CPSD plot of the acceleration, reveals that compared to PSO-tuned LQR, the proposed approach offers a significant reduction in vertical acceleration in both low- and high-frequency spectra, thereby considerably increasing passenger comfort. Moreover, to quantify the performance of the controller for both the road profiles 


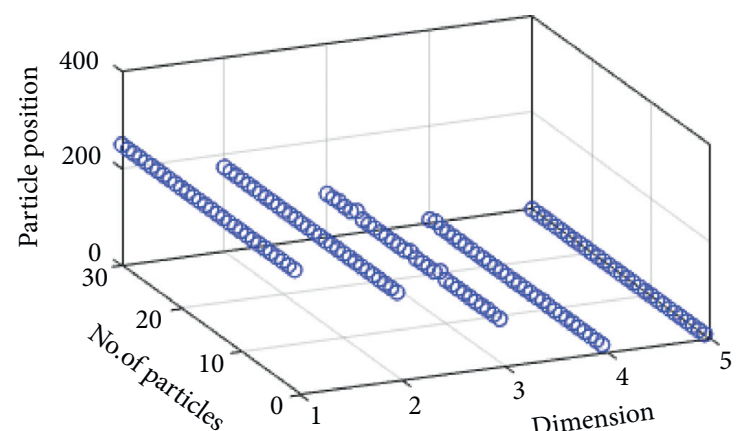

(a)

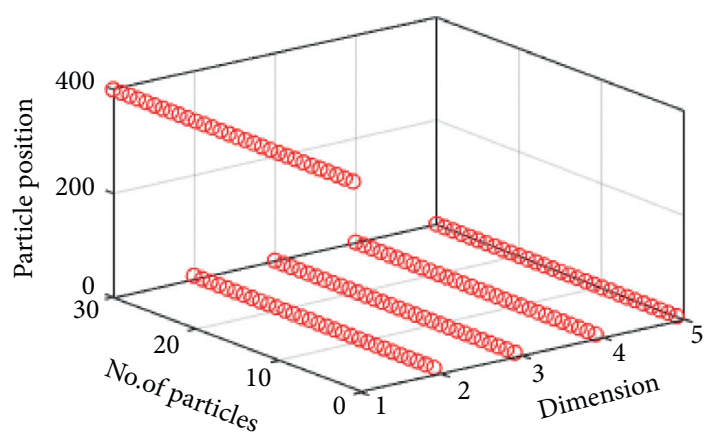

(b)

FIGURE 14: Particle convergence after 400 iterations. (a) PSO. (b) AIWSO.

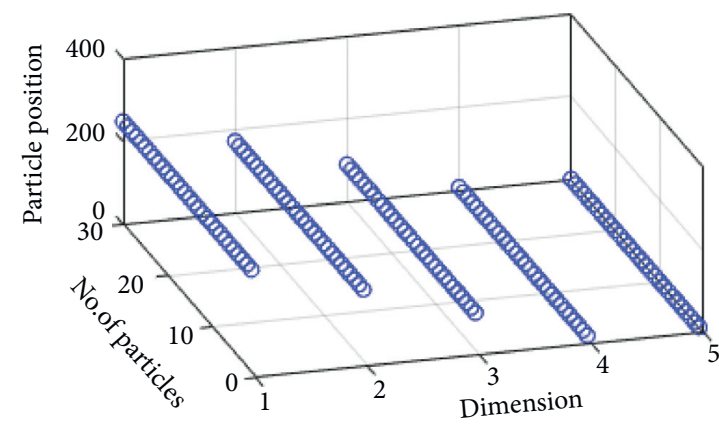

(a)

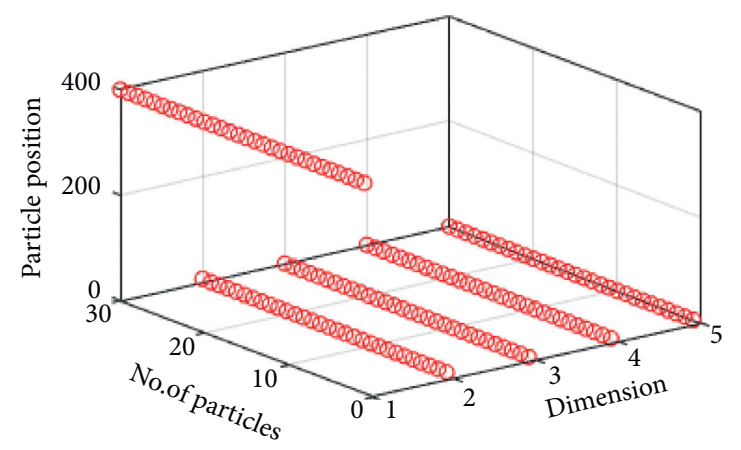

(b)

FIGURE 15: Particle convergence after 500 iterations. (a) PSO. (b) AIWPSO.

TABLe 3: Statistical analysis.

\begin{tabular}{lcc}
\hline Parameter & PSO & AIWPSO \\
\hline Mean & 0.0192 & 0.0148 \\
Standard deviation & 0.00086 & 0.00074 \\
Max & 0.182 & 0.0143 \\
Min & 0.02122 & 0.01545 \\
Range & 0.0032 & 0.0019 \\
\hline
\end{tabular}

TABLE 4: Weighting matrices of LQR.

\begin{tabular}{lcc}
\hline Parameter & PSO & AIWPSO \\
\hline \multirow{2}{*}{ Weighting matrices } & $Q=\operatorname{diag}(246.45,172.49,90.12,8.29)$ & $Q=\operatorname{diag}(38.23,92.46,21.12,79.76)$ \\
& $R=0.0319$ & $R=0.00312$ \\
\hline
\end{tabular}

TABle 5: Performance indices for ASS.

\begin{tabular}{lcccc}
\hline Road profile & Controller & RMS $\left(\mathrm{m} / \mathrm{s}^{2}\right)$ & FWRMS $\left(\mathrm{m} / \mathrm{s}^{2}\right)$ & 0.019 \\
Trapezoidal & PSO-LQR & 0.032 & 0.012 & 0.039 \\
& AIWPSO-LQR & 0.018 & 0.256 & 0.027 \\
\hline \multirow{2}{*}{ Sine-bump } & PSO-LQR & 0.413 & 0.214 & 0.801 \\
& AIWPSO-LQR & 0.291 & 0.598 \\
\hline
\end{tabular}




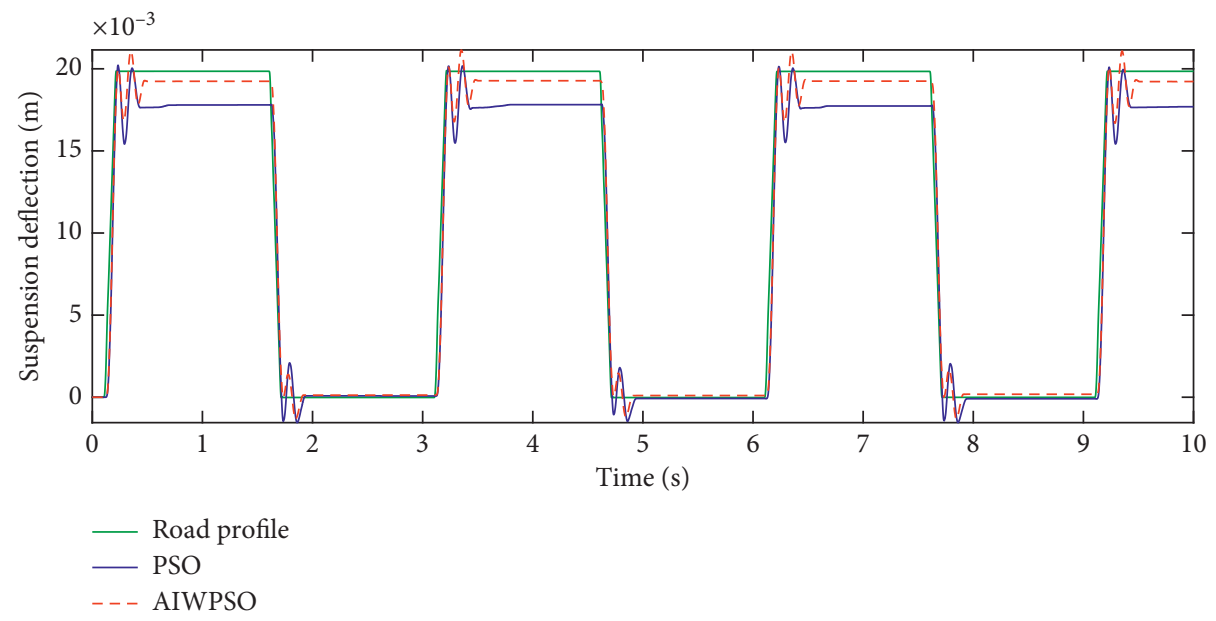

Figure 16: Suspension deflection for a trapezoidal profile.

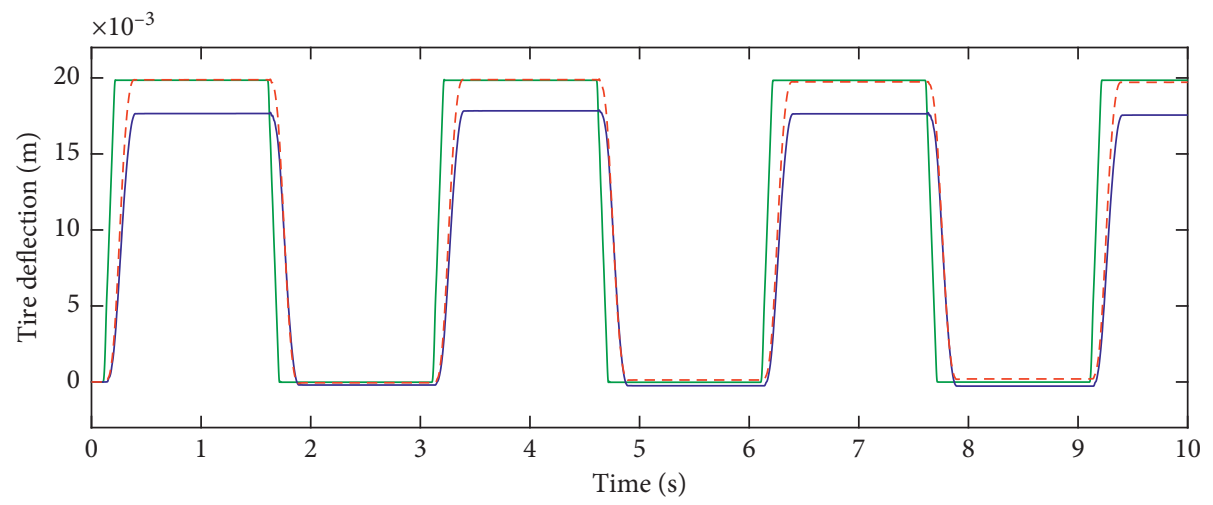

— Road profile

- PSO

- - - AIWPSO

Figure 17: Tire deflection for a trapezoidal profile.

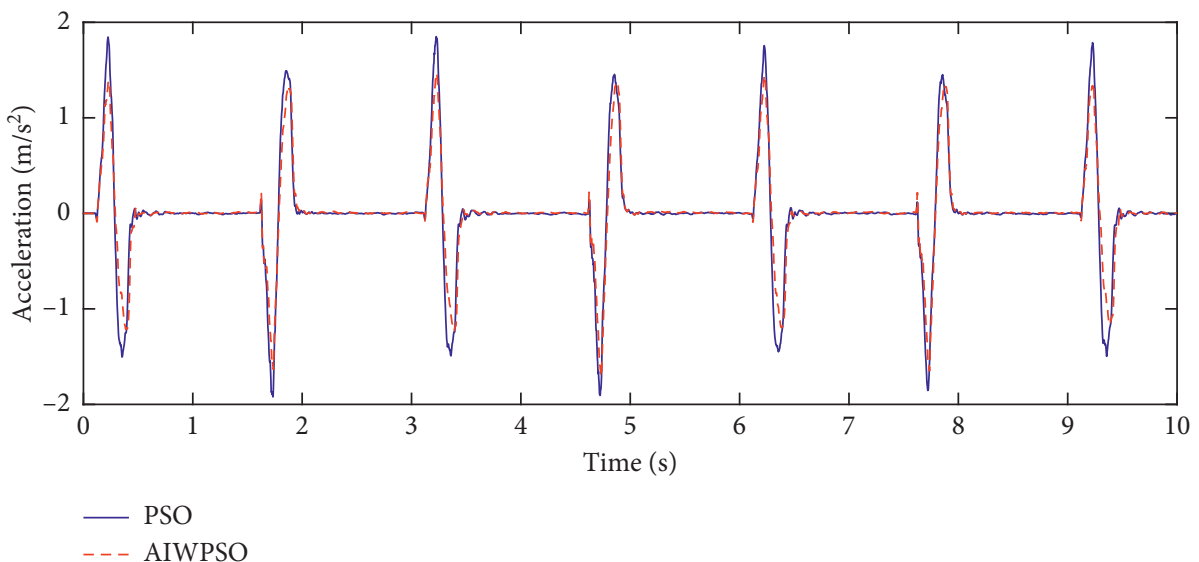

FIgURE 18: Acceleration of the vehicle body for a trapezoidal profile. 


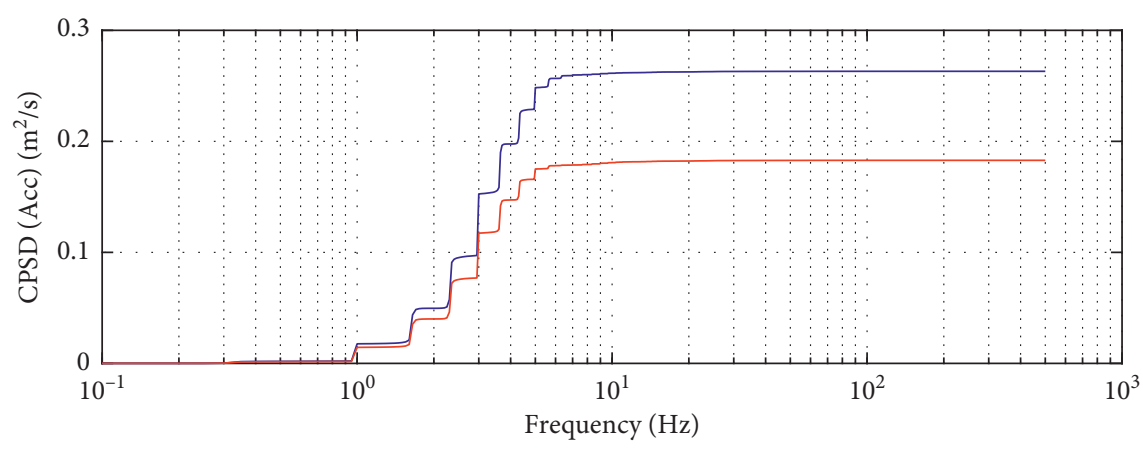

PSO

— AIWPSO

Figure 19: CPSD of acceleration for a trapezoidal profile.

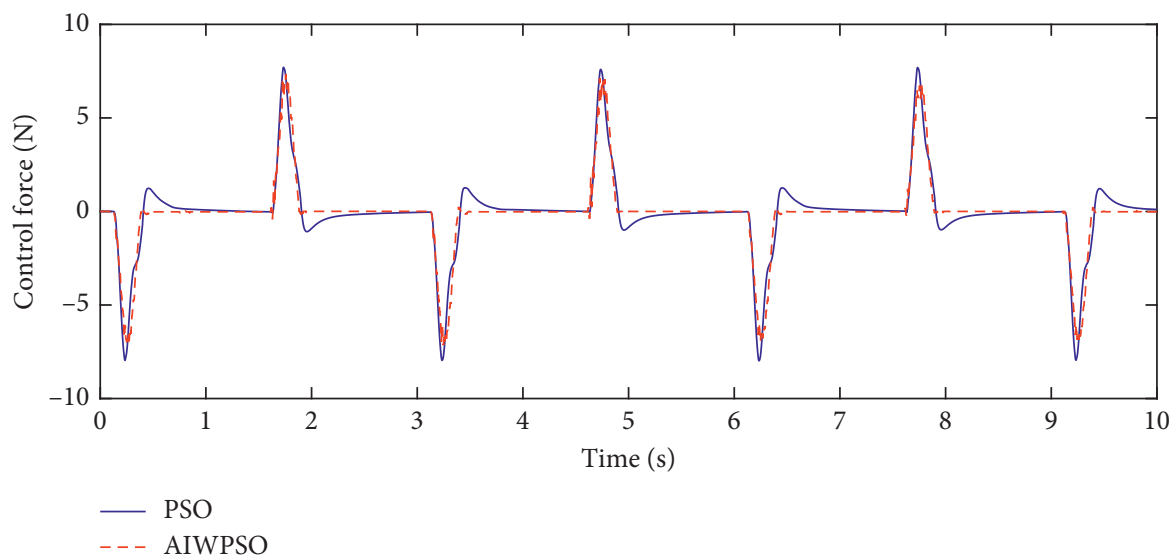

FIGURE 20: Actuator force for a trapezoidal profile.

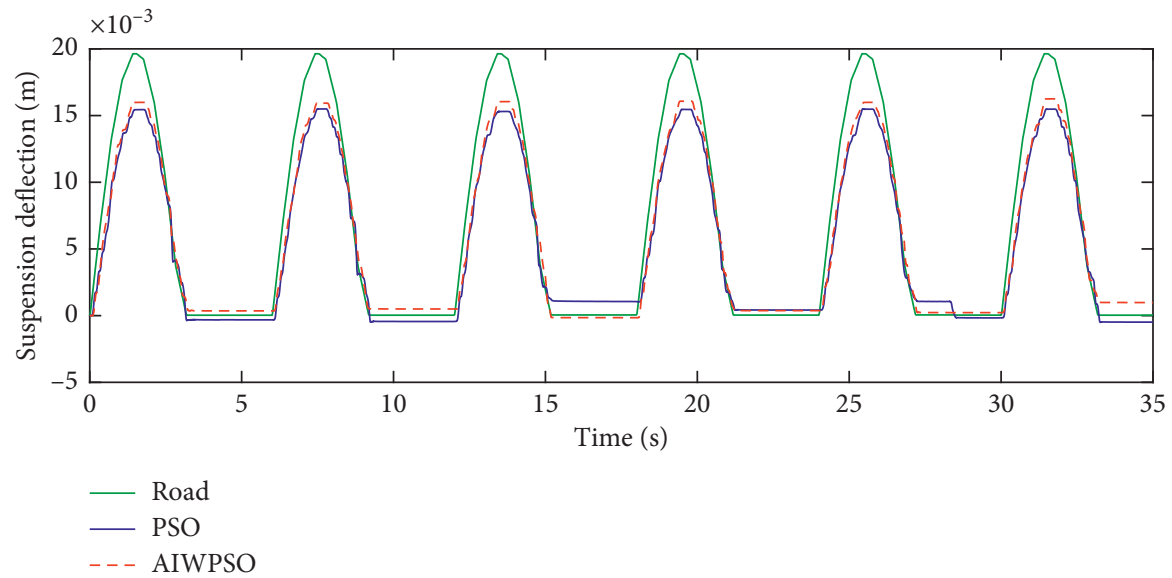

FIgURE 21: Suspension deflection for a bumpy road profile.

considered for evaluation, we compute three performance measures based on the ISO 2361-1 standards, which provide a guide to evaluate the human exposure to whole-body vibrations [31, 32]. The three performance metrics considered are root means square (RMS) of suspension travel, frequency-weighted RMS (FWRMS) of acceleration, and vibration dose value (VDV), which are mathematically described as follows: 


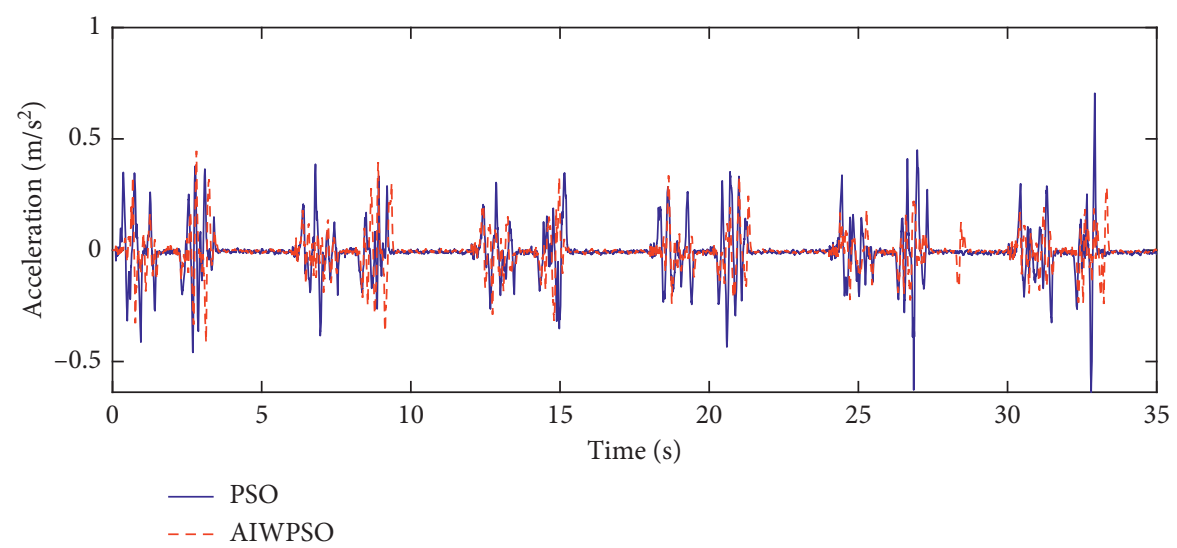

FIgURE 22: Vehicle body acceleration for a bumpy road profile.

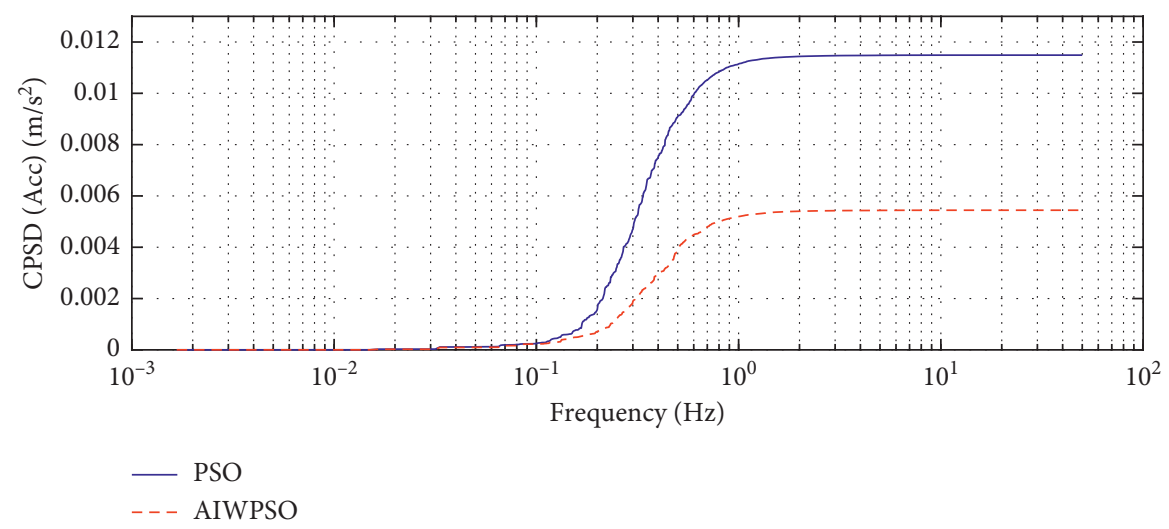

FIGURE 23: CPSD of acceleration for a bumpy road profile.

$$
\begin{aligned}
\text { RMS suspension travel } & =\left\{\frac{1}{T} \int_{0}^{T}\left[Z_{s}(t)-Z_{\mathrm{us}}(t)\right]^{2} \mathrm{~d} t\right\}^{(1 / 2)}, \\
A_{\mathrm{wh}}=\left\{\frac{1}{T} \int_{0}^{T}\left[a_{\mathrm{wh}}\right]^{2} \mathrm{~d} t\right\}^{(1 / 2)}, & \\
\operatorname{VDV} & =\left\{\int_{0}^{T}\left[a_{\mathrm{wh}}\right]^{4} \mathrm{~d} t\right\}^{(1 / 4)},
\end{aligned}
$$

where $a_{\text {wh }}$ is the frequency-weighted acceleration. Table 5 gives the performance metrics of the control schemes for the two road profiles. For a trapezoidal road profile, compared to conventional PSO-tuned LQR, the AIWPSO-tuned LQR reduces the FWRMS of body acceleration by $36.8 \%$ and the VDV by $30.76 \%$. Similarly, for a bumpy road excitation, the proposed scheme reduces the FWRMS and VDV by $16.4 \%$ and $25.34 \%$, respectively, compared to the conventional PSO-tuned LQR. These metrics highlight that the proposed scheme significantly improves not only the passenger comfort but also passenger safety by guaranteeing firm contact of the tire with the road surface.

\section{Conclusions}

To solve the weight optimization problem of LQR applied to a vehicle suspension system, this paper has presented an improved PSO which utilizes the adaptive inertia weight scheme.
The LQR weight selection problem is formulated as an optimization problem and the AIWPSO is utilized to find the optimal weights. Even though PSO is a robust optimization algorithm, it fails to draw a balance between the exploration and exploitation of the particles in search of the global optimum. Moreover, in the conventional PSO, the particles get trapped in the local optima, resulting in loss of diversity. Hence, to solve these two fundamental issues with PSO, an adaptive inertia weight is employed so that the velocity of the particles can be updated using the success rate as a feedback parameter. The statistical analysis of the optimal solutions highlights that compared to the conventional PSO, the AIWPSO can not only improve the convergence but also enhance the repeatability. The efficacy of the proposed scheme is experimentally validated on a quarter-car ASS for practical a road profile. The experimental results corroborate that the proposed scheme considerably improves passenger comfort and road handling.

\section{Data Availability}

The data used to support the findings of this study are included within the article.

\section{Conflicts of Interest}

The authors declare that there are no conflicts of interest regarding the publication of this paper. 


\section{Supplementary Materials}

The supplementary materials include HIL testing of the active suspension system. (Supplementary Materials)

\section{References}

[1] I. Fialho and G. J. Balas, "Road adaptive active suspension design using linear parameter-varying gain-scheduling," IEEE Transactions on Control Systems Technology, vol. 10, no. 1, pp. 43-54, 2002.

[2] X. Shao, F. Naghdy, and H. Du, "Reliable fuzzy $H \infty$ control for active suspension of in-wheel motor driven electric vehicles with dynamic damping," Mechanical Systems and Signal Processing, vol. 87, pp. 365-383, 2017.

[3] V. S. Deshpande, B. Mohan, P. D. Shendge, and S. B. Phadke, "Disturbance observer based sliding mode control of active suspension systems," Journal of Sound and Vibration, vol. 333, no. 11, pp. 2281-2296, 2014.

[4] J. Lin, R.-J. Lian, C.-N. Huang, and W.-T. Sie, "Enhanced fuzzy sliding mode controller for active suspension systems," Mechatronics, vol. 19, no. 7, pp. 1178-1190, 2009.

[5] J. Cao, P. Li, and H. Liu, "An interval fuzzy controller for vehicle active suspension systems," IEEE Transactions on Intelligent Transportation Systems, vol. 11, no. 4, pp. 885-895, 2010.

[6] H. Arefkhani, S. H. Sadati, and M. Shahravi, "Satellite attitude control using a novel constrained magnetic linear quadratic regulator," Control Engineering Practice, vol. 101, Article ID 104466, 2020.

[7] M. Habib, F. Khoucha, and A. Harrag, "GA-based robust LQR controller for interleaved boost DC-DC converter improving fuel cell voltage regulation," Electric Power Systems Research, vol. 152, pp. 438-456, 2017.

[8] Z. Han, N. Xu, H. Chen, Y. Huang, and B. Zhao, "Energyefficient control of electric vehicles based on linear quadratic regulator and phase plane analysis," Applied Energy, vol. 213, pp. 639-657, 2018.

[9] M. Mahmoodabadi and N. R. Babak, "Robust fuzzy linear quadratic regulator control optimized by multi-objective high exploration particle swarm optimization for a 4 degree-of-freedom quadrotor," Aerospace Science and Technology, vol. 97, pp. 1-13, 2020.

[10] L. Wang, H. Ni, W. Zhou, P. M. Pardalos, J. Fang, and M. Fei, "MBPOA-based LQR controller and its application to the double-parallel inverted pendulum system," Engineering Applications of Artificial Intelligence, vol. 36, pp. 262-268, 2014.

[11] K. Hassani and W.-S. Lee, "Multi-objective design of state feedback controllers using reinforced quantum-behaved particle swarm optimization," Applied Soft Computing, vol. 41, pp. 66-76, 2016.

[12] B. Ufnalski, A. Kaszewski, and L. M. Grzesiak, "Particle swarm optimization of the multioscillatory LQR for a three-phase four-wire voltage-source inverter with an LC output filter," IEEE Transactions on Industrial Electronics, vol. 62, no. 1, pp. 484-493, 2014.

[13] S.-J. Tsai, C.-L. Huo, Y.-K. Yang, and T.-Y. Sun, "Variable feedback gain control design based on particle swarm optimizer for automatic fighter tracking problems," Applied Soft Computing, vol. 13, no. 1, pp. 58-75, 2013.

[14] S. B. Karanki, M. K. Mishra, and B. K. Kumar, "Particle swarm optimization-based feedback controller for unified power-quality conditioner," IEEE Transactions on Power Delivery, vol. 25, no. 4, pp. 2814-2824, 2010.

[15] H. B. Duan and S. Q. Liu, "Non-linear dual-mode receding horizon control for multiple unmanned air vehicles formation flight based on chaotic particle swarm optimisation," IET Control Theory \& Applications, vol. 4, no. 11, pp. 2565-2578, 2010.

[16] Z. Qi, Q. Shi, and H. Zhang, “Tuning of digital PID controllers using particle swarm optimization algorithm for a CAN-based DC motor subject to stochastic delays," IEEE Transactions on Industrial Electronics, vol. 67, no. 7, pp. 5637-5646, 2019.

[17] K. D. Sharma, A. Chatterjee, and A. Rakshit, "A PSO-Lyapunov hybrid stable adaptive fuzzy tracking control approach for vision-based robot navigation," IEEE Transactions on Instrumentation and Measurement, vol. 61, no. 7, pp. 1908-1914, 2012.

[18] N. D. Zorić, A. M. Simonović, Z. S. Mitrović, and S. N. Stupar, "Optimal vibration control of smart composite beams with optimal size and location of piezoelectric sensing and actuation," Journal of Intelligent Material Systems and Structures, vol. 24, no. 4, pp. 499-526, 2013.

[19] X. Min, Y. Li, and S. Tong, "Adaptive fuzzy output feedback inverse optimal control for vehicle active suspension systems," Neurocomputing, vol. 403, pp. 257-267, 2020.

[20] G. P. S. and M. M. K., "A contemporary adaptive air suspension using LQR control for passenger vehicles," ISA Transactions, vol. 93, pp. 244-254, 2019.

[21] S. Kanarachos, A. M. Dizqah, G. Chrysakis, and M. E. Fitzpatrick, "Optimal design of a quadratic parameter varying vehicle suspension system using contrast-based fruit fly optimisation," Applied Soft Computing, vol. 62, pp. 463477, 2018.

[22] I. Damaj, M. Elshafei, M. El-Abd, and M. E. Aydin, "An analytical framework for high-speed hardware particle swarm optimization," Microprocessors and Microsystems, vol. 72, Article ID 102949, 2020.

[23] Q. Kang and H. He, "A novel discrete particle swarm optimization algorithm for meta-task assignment in heterogeneous computing systems," Microprocessors and Microsystems, vol. 35, no. 1, pp. 10-17, 2011.

[24] J. Tao, G. Sun, X. Wang, and L. Guo, "Robust optimization for a wing at drag divergence mach number based on an improved PSO algorithm," Aerospace Science and Technology, vol. 92, pp. 653-667, 2019.

[25] M. Shandilya, S. S. Pawar, and V. Chaurasia, "Design and optimization of a non-cross feed printed log periodic dipole array antenna using particle swarm optimization," AEU-International Journal of Electronics and Communications, vol. 93, pp. 172-181, 2018.

[26] D. H. Tungadio, J. A. Jordaan, and M. W. Siti, "Power system state estimation solution using modified models of PSO algorithm: comparative study," Measurement, vol. 92, pp. 508-523, 2016.

[27] R. C. Eberhart and Y. Shi, "Comparing inertia weights and constriction factors in particle swarm optimization," in Proceedings of the 2000 Congress on Evolutionary Computation, vol. 1, pp. 84-88, La Jolla, CA, USA, July 2000.

[28] A. Nickabadi, M. M. Ebadzadeh, and R. Safabakhsh, "A novel particle swarm optimization algorithm with adaptive inertia weight," Applied Soft Computing, vol. 11, no. 4, pp. 3658-3670, 2011.

[29] E. V. Kumar, G. S. Raaja, and J. Jerome, "Adaptive PSO for optimal LQR tracking control of 2 DoF laboratory helicopter," Applied Soft Computing, vol. 41, pp. 77-90, 2016. 
[30] M. Taherkhani and R. Safabakhsh, "A novel stability-based adaptive inertia weight for particle swarm optimization," Applied Soft Computing, vol. 38, pp. 281-295, 2016.

[31] M. P. Nagarkar, M. El-Gohary, Y. J. Bhalerao, G. J. V. Patil, and R. N. Z. Patil, "Artificial neural network predication and validation of optimum suspension parameters of a passive suspension system," SN Applied Sciences, vol. 1, no. 6, pp. 569-576, 2019.

[32] M. P. Nagarkar, G. J. Vikhe Patil, and R. N. Zaware Patil, "Optimization of nonlinear quarter car suspension-seatdriver model," Journal of Advanced Research, vol. 7, no. 6, pp. 991-1007, 2016. 